\title{
Antibiotic susceptibility pattern among clinical isolates of staphylococcus aureus with special reference to vancomycin
}

\author{
Usha M. G1, Shwetha D C ${ }^{2, *}$ \\ ${ }^{1}$ Professor, ${ }^{2}$ Assistant Professor, Dept. of Microbiology, ${ }^{1} \mathrm{JJM}$ Medical College, Davangere, Karnataka, ${ }^{2}$ Adichunchanagiri \\ Institute of Medical Sciences B.G. Nagara, Karnataka, India \\ *Corresponding Author: Shwetha D C \\ Email: shwetha.dasarahalli@gmail.com
}

Received: $1^{\text {st }}$ August, 2018

Accepted: $26^{\text {th }}$ September, 2018

\begin{abstract}
Introduction: Staphylococcus is a major pathogen of community and hospital acquired infections. Vancomycin is used in MRSA caused infections. Emergence of VISA and VRSA has been of great concern in clinical aspects.

Materials and Methods: All clinical samples were processed in the laboratory according to standard procedure. Inoculated plates were incubated at $37^{\circ} \mathrm{C}$ for $24-48$ hours. Only $S$. aureus isolates were included in the present study. Antibiotic susceptibility testing was done by Kirby-Bauer disc diffusion method using a panel of drugs. Cefoxitin disc was used to identify methicillin resistance. The MIC of vancomycin for MRSA isolates was carried out by Agar dilution method and E-test according to standard methods. Heteroresistance to vancomycin was detected by using BHI screen agar.

Results: $190 \mathrm{~S}$. aureus were isolated from various clinical samples. Most of the isolates were resistant to amoxyclav (96.2\%) followed by ciprofloxacin (84.2\%), erythromycin (33.2\%), Clindamycin (31.2\%), Cotrimoxazole (14.6\%), Teicoplanin (4.2\%), Mupirocin (2.1\%) and none of the isolates were resistant to linezolid. Out of 190 S. aureus isolates, 97 (51.1\%) were identified as MRSA. None of the isolates were resistant to vancomycin by agar dilution method and E-test method. Four out of 97 (4.1\%) MRSA isolates showed intermediate susceptibility to vancomycin. Among the isolates with MIC of $2 \mu \mathrm{g} / \mathrm{ml}, 5(19.2 \%)$ showed heteroresistance to vancomycin by BHI screen agar method.

Conclusion: Rapid identification of patients harboring VRSA, VISA or hVISA and adherence to infection control protocols are very important in controlling the dissemination of these pathogens.
\end{abstract}

Keywords: MRSA, VISA, VRSA, Heterointermediate resistant Staphylococcus aureus (hVISA), Agar dilution method, E-test.

\section{Introduction}

Staphylococcus aureus (S. aureus) is an opportunistic pathogen and the most common cause of nosocomial infections [Seivert DM et al., 2008]. Infections caused by $S$. aureus are difficult to treat as they have the ability to destroy neutrophils and also show antibiotic resistance [Ng ST et al., 2011]. During 1970 's, resistance to new semisynthetic penicillinase resistant antimicrobial agents (methicillin, oxacillin, nafcillin) developed [B Michael et al., 1996]. MRSA has been considered as a major nosocomial pathogen in health care facilities, but in the last decade it has also been observed to be an emerging pathogen in community acquired infections [Seralathan SE et al., 2017]. This led to the increased reliance on vancomycin for the treatment of Methicillin resistant S. aureus (MRSA) infections [Hageman JC et al., 2006]. In 1997, the first clinical isolate of $S$. aureus with reduced susceptibility to vancomycin was reported from Japan [Tenover FC et al., 2001 \& Tenover FC et al., 1998]. Subsequently, Vancomycin intermediate $S$. aureus (VISA) were reported in the United States and around the world [Tenover FC et al., 2007]. In June 2002, a Vancomycin resistant $S$. aureus (VRSA) was identified [Chang S et al., 2003].

There is an increasing number of strains of $S$. aureus showing heterointermediate resistance to vancomycin (hVISA). hVISA strains are defined as strains of $S$. aureus that contain subpopulation of vancomycin intermediate daughter cells [Song JH et al., 2004]. Hence, the present study was done to determine antibiotic susceptibility, possible presence of VISA and VRSA and also the presence of hVISA strains among vancomycin susceptible strains of $S$. aureus.

\section{Materials and Methods}

The present study was done after obtaining the institutional ethical committee clearance.

All clinical samples were processed in the laboratory according to standard procedure (samples were inoculated on blood agar and MacConkey agar except urine sample, which was inoculated on CLED medium).

Inoculated plates were incubated at $37^{\circ} \mathrm{C}$ for $24-48$ hours aerobically. Identification of isolate as $S$. aureus was done according to standard methods [Baird D et al., 1996] and only S. aureus isolates were included in the present study.

One hundred and ninety $S$. aureus were isolated from various clinical samples like pus, sputum, blood, urine, ear swab, pleural fluid, CSF, nasal swab and suction tip etc.

Antibiotic susceptibility testing was done by using Muller-Hinton agar by Kirby-Bauer disc diffusion method using a panel of drugs like Amoxyclav $(30 \mu \mathrm{g})$, ciprofloxacin $(5 \mu \mathrm{g})$, erythromycin $(15 \mu \mathrm{g})$, Clindamycin 
$(2 \mu \mathrm{g})$, Cotrimoxazole $(25 \mu \mathrm{g})$, Teicoplanin $(30 \mu \mathrm{g})$, Mupirocin $(5 \mu \mathrm{g})$, Linezolid $(30 \mu \mathrm{g})$. Cefoxitin disc $(30 \mu \mathrm{g})$ was used to identify methicillin resistance. $S$. aureus ATCC 25923 was used as a control strain.

The MIC of vancomycin for MRSA isolates was carried out by agar dilution method and E-test according to the standard methods.

Agar Dilution Method: Gradient plates of MHA plates were prepared with vancomycin $(0.5-32 \mu \mathrm{g} / \mathrm{ml}) .0 .5$ MacFarland matched culture suspension was 1 in 100 diluted with sterile saline and about $10 \mu \mathrm{l}$ was spot inoculated. Plates were incubated at $35^{\circ} \mathrm{C}$ for 24 hours. Lowest concentration of vancomycin that inhibited the visible growth of the strain was taken as MIC for that particular isolate.

E-test: E-test was done according to manufacturer's instructions. 0.5 MacFarland matched inoculum was swabbed on Muller-Hinton agar and E-strip was placed. Plates were incubated at $35^{\circ} \mathrm{C}$ for 24 hours. Vancomycin susceptible S. aureus ATCC 29213 was used as a control.

Isolate with MIC of $2 \mu \mathrm{g} / \mathrm{ml}$ were considered for heteroresistance detection. Heteroresistance to vancomycin was detected by using BHI screen agar.

BHI Screen Agar Plates: BHI screen agar was prepared in-house by adding $4 \mu \mathrm{g} / \mathrm{ml}$ of vancomycin and $16 \mathrm{~g} / \mathrm{l}$ pancreatic digest of casein. Four $10 \mu \mathrm{l}$ droplets from 0.5 MacFarland suspension were dropped by a pipette onto the BHI screen agar plates, allowed to dry for $5 \mathrm{~min}$ and incubated at $35^{\circ} \mathrm{C}$. Plates were examined at 24 hours and 48 hours. An isolate was considered hVISA if at least one droplet had two or more colonies [Satola SW et al., 2011].

\section{Results}

190 S. aureus were isolated from various clinical samples like pus (126), blood (16), sputum (12), urine (12), nasal swab (08), suction tip (05), ear swab (03), endotracheal tube (03), bronchoalveolar lavage (02), pleural fluid (02) and CSF (01) (Table 1).

Most of the isolates were resistant to amoxyclav (96.2\%) followed by ciprofloxacin (84.2\%), erythromycin (33.2\%), Clindamycin (31.2\%), Cotrimoxazole (14.6\%), Teicoplanin (4.2\%), Mupirocin $(2.1 \%)$ and none of the isolates were resistant to Linezolid (Table 2).

Out of 190 S. aureus isolates, $97(51.1 \%)$ were identified as MRSA. None of the isolates were resistant to vancomycin by agar dilution method and E-test method. Four out of 97 (4.1\%) MRSA isolates showed intermediate susceptibility to vancomycin by both agar dilution method and E-test.

Among the isolates with MIC of $2 \mu \mathrm{g} / \mathrm{ml}, 5(19.2 \%)$ showed heteroresistance to vancomycin by BHI screen agar method.
Table 1: Isolation of $S$. aureus from various clinical samples

\begin{tabular}{|l|c|}
\hline Sample & $\begin{array}{c}\text { Number of S. aureus } \\
\text { isolates }\end{array}$ \\
\hline Pus & 126 \\
\hline Blood & 16 \\
\hline Sputum & 12 \\
\hline Urine & 12 \\
\hline Nasal swab & 08 \\
\hline Suction tip & 05 \\
\hline Ear swab & 03 \\
\hline Endotracheal tube & 03 \\
\hline Pleural fluid & 02 \\
\hline Bronchoalveolar lavage & 02 \\
\hline Cerebrospinal fluid & 01 \\
\hline Total & 190 \\
\hline
\end{tabular}

Totally 190 isolates of S.aureus from different samples were included. Samples from which bacteria other than S.aureus isolated were not included in the study.

Table 2: Antibiotic susceptibility pattern of $S$. aureus isolates

\begin{tabular}{|l|c|c|}
\hline \multicolumn{1}{|c|}{ Antibiotic } & $\begin{array}{c}\text { Number of } \\
\text { resistant strains } \\
\text { Out of 190 }\end{array}$ & $\begin{array}{c}\text { Percentage of } \\
\text { resistance }\end{array}$ \\
\hline Amoxyclav & 185 & 96.2 \\
\hline Ciprofloxacin & 162 & 84.2 \\
\hline Erythromycin & 64 & 33.2 \\
\hline Clindamycin & 60 & 31.2 \\
\hline Co-trimoxazole & 28 & 14.6 \\
\hline Teicoplanin & 08 & 04.2 \\
\hline Mupirocin & 04 & 02.1 \\
\hline Linezolid & None & None \\
\hline
\end{tabular}

\section{Discussion}

S. aureus is an important cause of community and hospital infections [Saravanan $M$ et al., 2013]. Antibiotic resistant staphylococci, especially MRSA have become the most common cause of hospital acquired infections worldwide. MRSA is of concern, not only because of its resistance to methicillin, but also because of its general resistance to many other chemotherapeutic agents.

Therapeutic options for MRSA infections are limited to a very expensive and potentially toxic drugs like teicoplanin, vancomycin, linezolid and daptomycin etc [K Jayatilleke et al., 2012]. So, early detection of MRSA and formulation of effective antibiotic therapy, along with infection control is very important from an epidemiological view point. The present study showed the MRSA prevalence of $51.1 \%$ among clinical samples, which is comparable to the results of other studies [K Jayatilleke et al., 2012, Joshi S et al., 2013 \& Bukhari SZ et al., 2011]. Cefoxitin disc diffusion method was done to screen MRSA strains because 
cefoxitin is a potent inducer of mecA gene, gives clearer endpoints, less affected by the hyperproduction of penicillinase, requires no special medium or incubation temperature [TA Dhanalakshmi et al., 2012].

In our study, most of the isolates were resistant to amoxyclav (96.2\%) followed by ciprofloxacin (84.2\%), erythromycin (33.2\%), Clindamycin $(31.2 \%)$ etc. This high prevalence of drug resistance may be due to the irrational antibiotic usage, due to its easy availability at the drug store without prescription, injudicious use in hospitals and uncontrolled use in agriculture, animal husbandry and fisheries [Tiwari HK et al., 2006].

In view of this antibiotic resistance, vancomycin has been the drug of last resort. Transfer of glycopeptide and macrolide resistant genes by transconjugation among Enterococci and from Enterococci fecalis to $S$. aureus have been reported [Saha B et al., 2008]. The present study showed decreased susceptibility to vancomycin. Four out of 97 (4.1\%) MRSA isolates showed intermediate susceptibility to vancomycin, but none of them showed resistance to vancomycin. In a study by Sharma $\mathrm{P}$ et al., 2012, 16 out of $156(11.5 \%)$ S. aureus isolates showed intermediate susceptibility to vancomycin and no VRSA were detected. Study by Saderi H et al., 2005 showed that $3.5 \%$ of the isolates were VRSA and another study by T.A. Dhanalakshmi et al., $2012 \mathrm{did}$ not report any case of VISA and VRSA. Though a very few instances only, VISA are a definite entity now. But the large scale development and subsequent spread of resistance to vancomycin is perceived as a fearsome threat to the already challenging therapy of MRSA [Assadullah S et al., 2003].

The present study revealed the waning susceptibility of staphylococci to vancomycin. This may be as a result of prolonged exposure of organisms to constant level of vancomycin in an opportune environment [Tenover FC et al., 1998].

Infections involving hVISA pose unique problem. Such strains are susceptible in vitro to vancomycin but contain subpopulation of 1 in $10^{6}$ cells that can grow in the presence of $\geq 4 \mu \mathrm{g} / \mathrm{ml}$ of vancomycin. Because of the increased reports of vancomycin treatment failures and poor outcomes of patients infected with hVISA, an accurate and practical method for the detection of hVISA is very important [Satola SW et al., 2011].

E-test macromethod is expensive and hVISA testing by population analysis is labor-intensive, time consuming and unsuitable for routine use [Fitzgibbon MM et al., 2007]. Hence, BHI screen agar was easy to perform and is used in the detection of hVISA. In the present study, $19.2 \%$ were hVISA strains.

\section{Limitation of the Study}

MecA gene detection by PCR technique, which is considered the gold standard method for MRSA detection was not done in our study due to economic constraints and also controls were not used in hetero resistance (hVISA) detection methods.

\section{Conclusion}

The present study reveals the emergence of $S$. aureus with decreased susceptibility to vancomycin and indicates the magnitude of antibiotic resistance in and around the study area. The major cause of this may be unawareness and indiscriminate use of broad-spectrum antibiotics. Rapid identification of patients harboring VRSA, VISA or hVISA as well as prompt isolation and adherence to infection control protocols are paramount in controlling the dissemination of these pathogens.

\author{
Abbreviations \\ MRSA- Methicillin resistant Staphylococcus aureus \\ VISA- Vancomycin intermediate Staphylococcus \\ aureus \\ VRSA- Vancomycin resistant Staphylococus aureus \\ hVISA- Heterointermediate resistant Staphylococcus \\ aureus \\ CLED- Cysteine lactose electrolyte deficient \\ CSF- Cerebrospinal fluid \\ ATCC-American type culture collection \\ MIC- Minimum inhibitory concentration \\ BHI- Brain heart infusion
}

\section{References}

1. Assadullah S, Kakru DK, Thoker MA, Bhat FA, Hussain N, Shah A. Emergence of low level vancomycin resistance in MRSA. Indian Journal of Medical Microbiology. 2003;21(3):196-8.

2. B Michael, P Richard, Pasculle AW. VancomycinResistant Staphylococcus aureus: Prospectives on Measures Needed for Control. Ann Intern Med. 1996;124(3):329-34.

3. Baird D. Staphylococci: Cluster-forming Gram-positive cocci In: Collee JG, Fraser AG, Marmion BP, Simmons A, Editors. Mackie \& McCartney Practical Medical Microbiology, 14th ed. (Churchill Livingston, New York). 1996:245-61.

4. Bukhari SZ, Ahmed S, Zia N. Antimicrobial susceptibility pattern of Staphylococcus aureus clinical isolates and efficacy of laboratory tests to diagnose MRSA: A multi-centre study. J Ayub Med Coll Abbottbad. 2011;23(1):139-42.

5. Chang S, Sievert DM, Hageman JC, Boulton ML, Tenover FC, Downes FP et al. Infection with Vancomycin-resistant Staphylococcus aureus Containing the VanA Resistance Gene. The New England Journal of Medicine. 2003;348:1342-7.

6. Fitzgibbon MM, Rossney AS, Connell BO. Investigation of Reduced Susceptibility to Glycopeptides among Methicillin-Resistant Staphylococcus aureus isolates from patients in Ireland and Evaluation of Agar screening Methods for Detection of Heterogeneously GlycopeptideIntermediate S.aureus. J Clin Microbiol. 2007;45(10):3263-69

7. Hageman JC, Patel JB, Carey RC, Tenover FC, McDonald LC. Investigation and control of Vancomycinintermediate and -resistant Staphylococcus aureus: A Guide for Health Departments and Infection Control Personnel. Atlanta, GA 2006. 
8. Joshi S, Ray P, Manchand V, Bajaj J, Chitnis DS, Gautam et al. Methicillin Resistant Staphylococcus aureus(MRSA) in India: Prevalence and susceptibility pattern. Indian J Med Res. 2013;137:363-69.

9. K Jayatilleke, P Bandara. Antibiotic sensitivity pattern of Staphylococcus aureus in a tertiary care hospital of Sri Lanka. Sri Lanka Journal of Infectious Diseases. 2012;2(2):13-17.

10. Ng ST, Lim CY, Tan CS, Abd Karim A, Haron H, Ahmad $\mathrm{N}$ et al. Emergence of Vancomycin-Resistant Staphylococcus aureus(VRSA). Webmed Central Infectious Diseases. 2011;2(12):WMC002787.

11. Saderi H, Owlia P, Shahrbanooie R. Vancomycin resistance among clinical isolates of Staphylococcus aureus. Archives of Iranian Medicine. 2005;8(2):100-103.

12. Saha B, Singh AK, Ghosh A, Bal M. Identification and characterization of a vancomycin resistant Staphylococcus aureus isolated from Kolkata (South Asia). J Med Microbiol. 2008;57:72-79.

13. Saravanan M, Nanda A, Tesfaye J. Antibiotic Susceptibility Pattern of Methertiary Hospital in Hosur, South India. American Journal of Microbiological Research. 2013;1(2):21-4.

14. Satola SW, Farley MM, Anderson KF, Patel JB. Comparison of Detection Methods for Heteroresistant Vancomycin Intermediate Staphylococcus aureus with the Population Analysis Profile Method as the Reference Method. J Clin Microbiol. 2011;49(1):177-183.

15. Seralathan SE, Sheeladevi C, Shashikala N, Saranathan, Prashanth K. Varying Drug Resistant Pattern of MRSA Isolates with PVL Gene. Int J Curr Microbiol App Sci. 2017;6(5):548-552.

16. Sharma P, Vishwanath G. Study of Vacomycin susceptibility in Methicillin resistant Staphylococcus aureus isolated from clinical samples. Annals of Tropical Medicine and Public Health. 2012;5(3):178-80.

17. Sievert DM, Rudrik JT, Patel JB, McDonald C, Wilkins MJ, Hageman JC et al. Vancomycin-Resistant Staphylococcus aureus in the United States, 2002-2006. Clin Infect Dis. 2008;46:668-74.
18. Song JH, Hiramatsu K, Suh JY, Ko KS, Ito J, Kapi M et al. Emergence in Asian Countries of Staphylocoocus aureus with Reduced susceptibility to Vancomycin. Antimicrobial agents and Chemotherapy. 2004;48(12):4926-28

19. TA Dhanalakshmi, BL Umapathy, DR Mohan. Prevalence of Methicillin, Vancomycin and multidrug resistance among Staphylococcus aureus. Journal of Clinical and Diagnostic Research. 2012;6(6):974-77.

20. Tenover FC, Biddle JW, Lancaster MV. Increasing Resistance to Vancomycin and other Glycopeptides in Staphylococcus aureus. Emerg Infect Dis. 2001;7(2):32732.

21. Tenover FC, Lancaster MV, Hill BC, Steward CD, Stocker SA, Hancock GA et al. Characterization of Staphylococci with Reduced Susceptibilities to Vancomycin and Other Glycopetides. J Clin Microbiol. 1998;36(4):1020-27.

22. Tenover FC, Moellering RC. The Rationale for Revising the Clinical and Laboratory Standards Institute Vancomycin Minimal Inhibitory Concentration Interpretive Criteria for Staphylococcus aureus. Clin Infect Dis. 2007;44:1208-5.

23. Tiwari HK, Sen MR. Emergence of vancomycin resistant Staphylococcus aureus from a tertiary care hospital from northern part of India. BMC Infectious Diseases. 2006;6:150.

How to cite this article: Usha M G, Shwetha D C. Antibiotic susceptibility pattern among clinical isolates of staphylococcus aureus with special reference to vancomycin. Indian J Microbiol Res. 2018;5(4):488-491. 